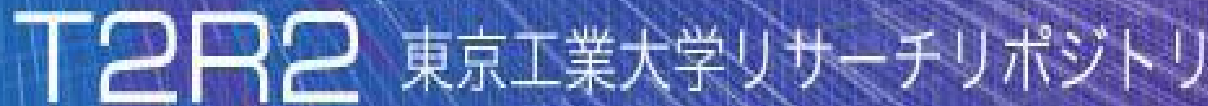

\section{Tokyo Tech Research Repository}

\section{論文 /著書情報 \\ Article /Book Information}

\begin{tabular}{|c|c|}
\hline Title & Development of Terrain Adaptive Sole for Multi-legged Walking Robot \\
\hline Author & Shumpei Ohtsuka, Gen Endo, Edwardo F. Fukushima, Shigeo Hirose \\
\hline Journal/Book name & , , , pp. 5354-5359 \\
\hline Issue date & 2010, 10 \\
\hline DOI & http://dx.doi.org/10.1109/RO S.2010.5649532 \\
\hline URL & http://www.ieee.org/ndex.html \\
\hline Copyright & $\begin{array}{l}\text { (c) } 2010 \text { IEEE. Personal use of this material is permitted. Permission } \\
\text { from IEEE must be obtained for all other users, including } \\
\text { reprinting/republishing this material for advertising or promotional } \\
\text { purposes, creating new collective works for resale or redistribution to } \\
\text { servers or lists, or reuse of any copyrighted components of this work in } \\
\text { other works. }\end{array}$ \\
\hline Note & $\begin{array}{l}\text { このファイルは著者 (最終) 版です。 } \\
\text { This file is author (final) version. }\end{array}$ \\
\hline
\end{tabular}




\title{
Development of Terrain Adaptive Sole for Multi-legged Walking Robot
}

\author{
Shumpei Ohtsuka, Gen Endo, Edwardo F. Fukushima and Shigeo Hirose
}

\begin{abstract}
Foot mechanism of walking robots needs to adapt to rough terrain enough because the walking robot is requested to have the ability to walk stably on rugged ground. However, the foot mechanism of multi-leg walking robots have been often modeled as a point foot or a disc sole so far, and there is a problem that the robot can not fully use the sole area as a support polygon on rough terrain. To solve the problem, the terrain adaptive sole is developed which can adapt to the ground with small ruggedness and support the ankle moment to acquire stable footholds on uneven terrain. The terrain adaptive sole is composed of a deformation mechanism, a shape-fixing mechanism and a vertical force sensor, which are developed for quadruped walking robot TITAN VIII. The deformation mechanism changes the shape of the foot according to the terrain and the shape-fixing mechanism fixes the shape after the foot landing. These functions are achieved by a passive mechanism to be robust and lightweight. We demonstrate that TITAN VIII with the proposed terrain adaptive sole can successfully negotiate a rough terrain.
\end{abstract}

\section{INTRODUCTION}

The legged robot is expected to be effective in an environment where wheel type and the crawler type robots are difficult to go, and various researches have been conducted [1]. The locomotion strategy of the legged robot in a rough terrain can be generally classified into two groups depending on the roughness of the terrain. If terrain undulation is very large compared with the size of the robot, we need to modify the trajectory of the legs and control the posture of the robot to negotiate such a large undulation, which usually requires a large calculation cost and takes longer time [2]. On the other hand, if terrain undulation is not so large, a mechanical adaptation by a sole mechanism is desirable to absorb the roughness of the terrain because it does not need to change the preplanned leg trajectory. A mechanical adaptation by a sole can simplify a gait control algorithm and provide the robot with faster locomotion.

In this context,we have considered the importance of optimum design of the foot mechanism, and conducted the study so far [3]. For example, the ankle mechanism that adopted a remote central mechanism was developed by Ogata [4]. It is based on the idea that the foot mechanism composed of a passive mechanism can be the lightweight and compact construction, support large load and achieve high speed adaptation according to the terrain. However, it was a design that valued the adaptability to the inclination of the terrain, and there was a problem in the adaptability to the ruggedness.

Shigeo Hirose is with Dept. of Mechano-Aerospace Engineering, Tokyo Institute of Technology, 2-12-1 O-okayama Meguro-ku Tokyo 152-8552, Japan hirose@mes.titech.ac.jp

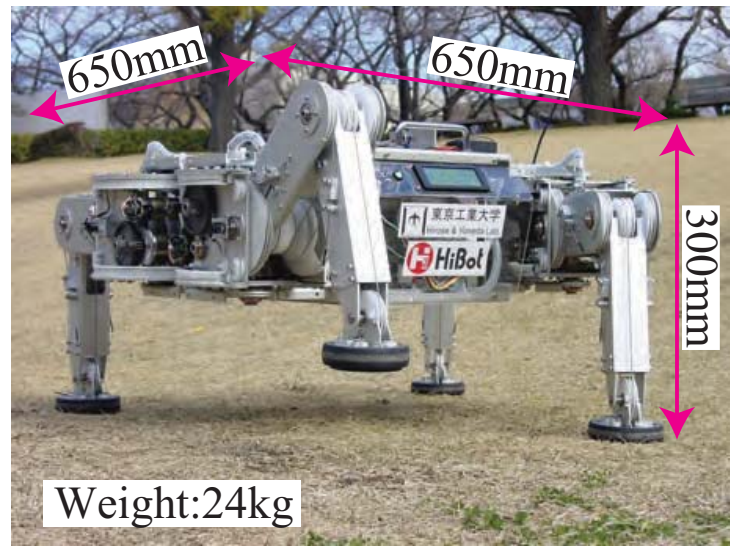

Fig. 1. TITAN VIII[6]

Semi-active type foot mechanism WS-5 whose shape of the foot is actively fixed by the installed actuator was developed by Hashimoto [5]. However it might be difficult to achieve quick adaptation, robustness, high resistance to environment due to shape-fixing actuation.

In this paper, the terrain adaptive sole that can adapt passively to the rough terrain including the bump and the inclination and secure a steady foothold is developed for the quadruped walking robot TITAN VIII (Fig.1). Our target terrain is a static and rigid environment where height undulation is up to $20 \mathrm{~mm}$. If the undulation is over $20 \mathrm{~mm}$, we modify the leg trajectory which is reported in Section IV.B.

\section{FUNCTIONS OF TERRAIN ADAPTIVE SOLE}

In some walking robots such as TITAN VIII, the improvement of the stability has been achieved by using the parallel linkage for the ankle joint which always keeps the relative angle of the sole parallel to the body so that the sole area can be used as support polygon. However, there was a problem that the sole which is composed of flat and hard plate is not able to adapt to the rough terrain. Thus the sole area is not made the best use of.

Moreover, from the viewpoint of reducing the weight of the foot, the foot mechanism of a multi- legged walking robot for adapting to the terrain tends to be simplified, so the foot mechanism is composed of a ball joint or a free universal joint [7]. Or they are even omitted and single point ground type mechanism is used as foot mechanism. Although quick adaptation to the terrain is possible, the sole area is not possible to use as a support polygon because it is not 


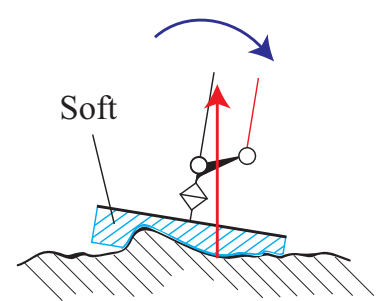

(a) without shape-fixing function

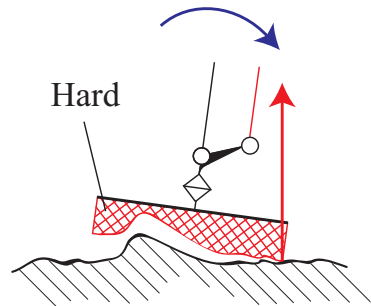

(b) with shape-fixing function

Fig. 2. Motion of sole mechanism
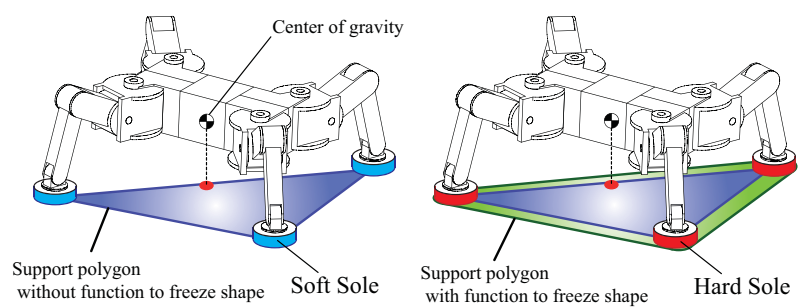

Fig. 3. Difference in support polygons

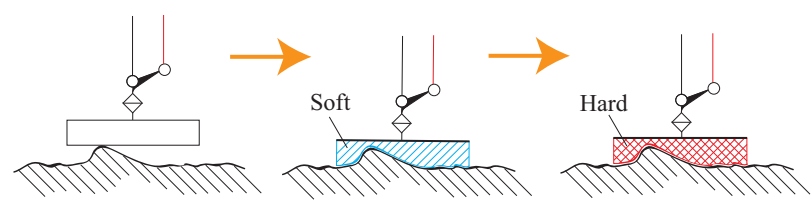

Fig. 4. Required functions for terrain adaptive sole

able to support the ankle moment while the supporting leg phase even if the sole area is enlarged (Fig.2,Fig.3).

From the above-mentioned, following two functions are thought to be necessary to support the ankle moment in the supporting leg phase and to construct a steady foothold on rough terrain (Fig.4).

1) Deformation function: function to deform the shape of the foot according to ruggedness when landing

2) Shape-fixing function: function to fix the shape of the foot deformed according to the ground after landing

A flat hard sole cannot deform along to a rough terrain so it is not possible to touch with full sole area. So, sole area is not able to be used as support polygon. To solve the problem, the function to deform the foot shape quickly according to the ruggedness of the terrain when landing is needed. However, when the force is applied to the foot mechanism which has only deforming function, the foot shape will deform and the foot can not generate the torque to prevent from falling. To solve the problem, it is thought that the function to fix the sole shape after deforming is necessary. Moreover, the passive mechanism which fulfills the above two functions not by actuators can achieve quick adaptation and a compact solid mechanism.

\section{DESIGN OF TERRAIN ADAPTIVE SOLE}

A prototype model of the terrain adaptive sole for quadruped walking robot TITAN VIII is shown in Fig.5

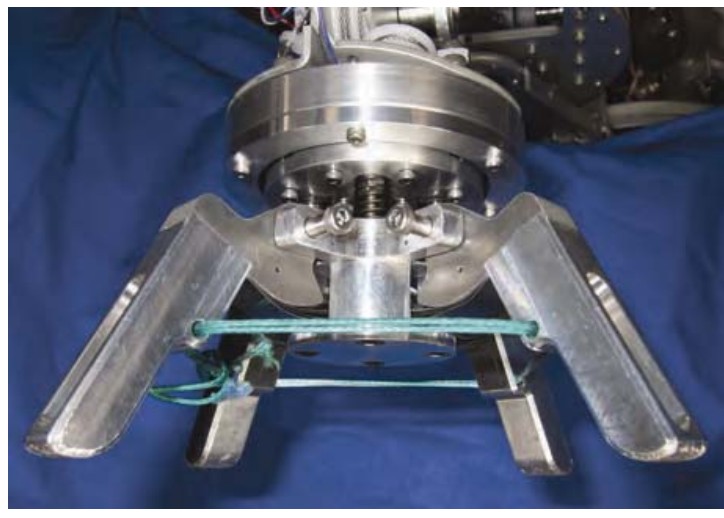

Fig. 5. Overview of terrain adaptive sole

TABLE I

SPECIFICATION OF TERRAIN ADAPTIVE SOLE

\begin{tabular}{|c|c|c|c|}
\hline & $\begin{array}{c}\text { Diameter of } \\
\text { sole area }\end{array}$ & Height & Weight \\
\hline Swing phase & \multirow{2}{*}{$\Phi 134 \mathrm{~mm}$} & $74 \mathrm{~mm}$ & \multirow{2}{*}{$330 \mathrm{~g}$} \\
\hline Support phase & & $70 \mathrm{~mm}$ & \\
\hline
\end{tabular}

and the parameters are shown in Table.I. Structure and mechanism are shown in Fig.6 and Fig.7.

The developed mechanism is composed of deformation mechanism, shape-fixing mechanism and vertical force sensor. Deformation mechanism consists of the finger mechanisms which are connected by a string. Shape-fixing mechanism fixes the relative motion of the finger mechanisms by suppressing the overlapped brake plates which are installed in the finger mechanism with a center rod after the landing. Vertical force sensor measures the displacement of the leaf spring with the micro photo sensor.

Adapting motion sequence is as follows; First, finger mechanisms connected with the string are pulled when a part of finger mechanism touches on the ground, and deforms according to the terrain shape (Fig.8(2)). Next, when all the finger mechanisms touch the ground, the string makes finger mechanism motion stopped, and the center rod supported with the leaf spring suppresses overlapped part of the brake sheets, then, motion of the finger mechanism is tightly locked, and sole shape is fixed (Fig.8(3)). In addition, the force which is applied to the center rod is measured with micro photo sensor by measuring the displacement of the leaf spring (Fig.8(3)).

\section{A. Deformation Mechanism}

Deformation mechanism is composed of the connected differential mechanism [8] that all the finger mechanisms are connected by a string (Fig.13). Each finger moves smoothly by a small force acting on the fingertips and the string connects all the finger mechanisms by passing through the hole of the finger mechanisms. This synchronized method by the simple structure permits the deformation mechanism to adapt to higher bump. Moreover, this deformation 


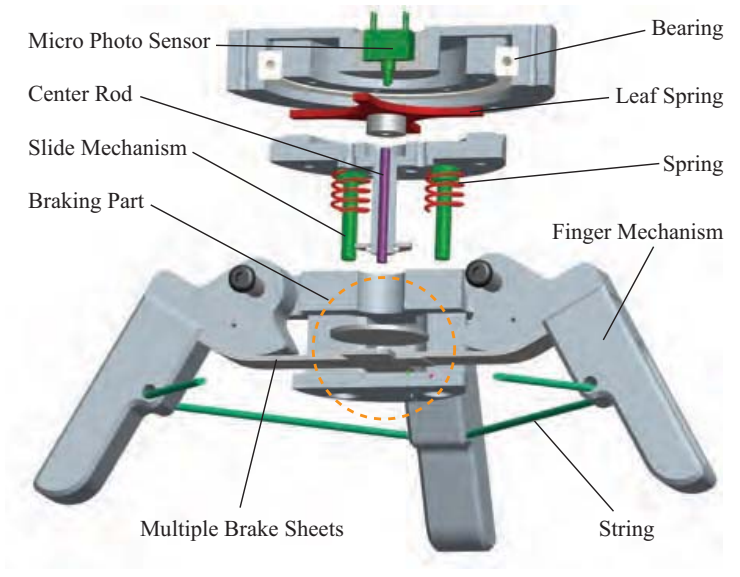

Fig. 6. Structure of terrain adaptive sole

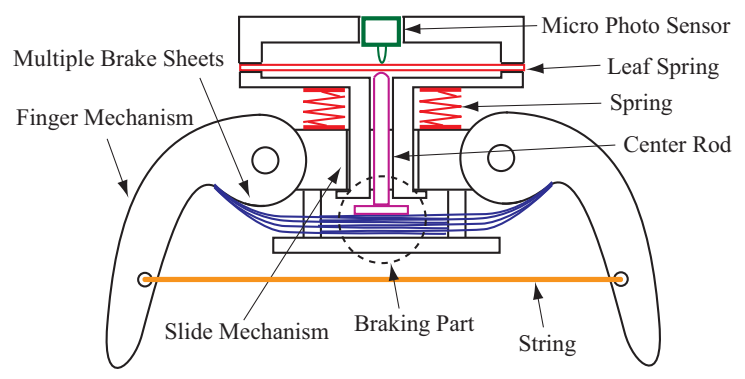

Fig. 7. Mechanism of terrain adaptive sole

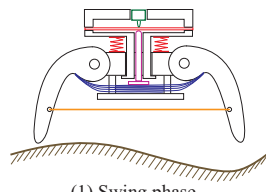

(1) Swing phase

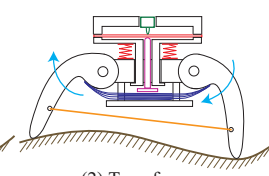

(2) Transform

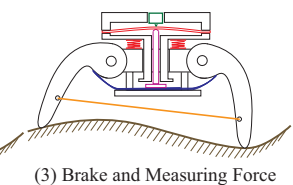

(3) Brake and Measuring Force
Fig. 8. Sequence of adapting motion of terrain adaptive sole

mechanism restricts relative motion of the fingers when all the finger mechanisms touch the ground, and after that the shape-fixing mechanism supported by the spring descends to press braking sheets. After pressing the braking sheets with sufficient vertical force, the shape of the sole is rigidly fixed.

In addition, on a flat terrain, the shape of the deformation mechanism doesn't change between the swing phase and the support phase due to the above-mentioned mechanism. This is advantageous because we can prevent the foot mechanism from interfering with the rugged ground when landing. And, difference between the height that foot actually goes up from the terrain surface and the height that the leg was raised by the robot is limited to only the motion of the slide mechanism.

\section{B. Shape-fixing Mechanism}

The shape-fixing mechanism is composed of multiple brake sheets made of SUS304 attached to the finger mechanism. The shape of the deformation mechanism is fixed by suppressing overlapping part of the brake sheets with

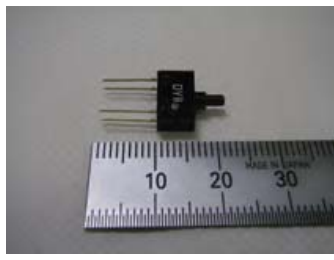

Fig. 9. Micro photo sensor
Fig. 10. Leaf spring
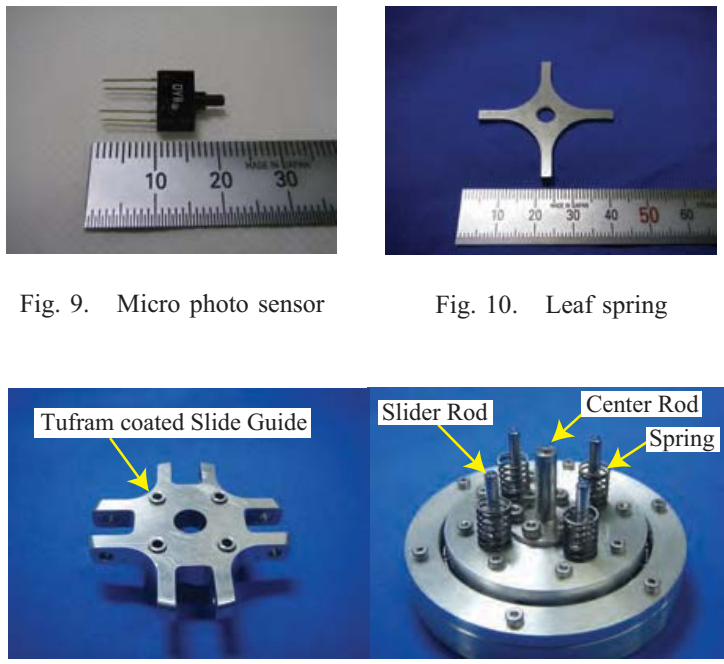

Fig. 11. Slide mechanism

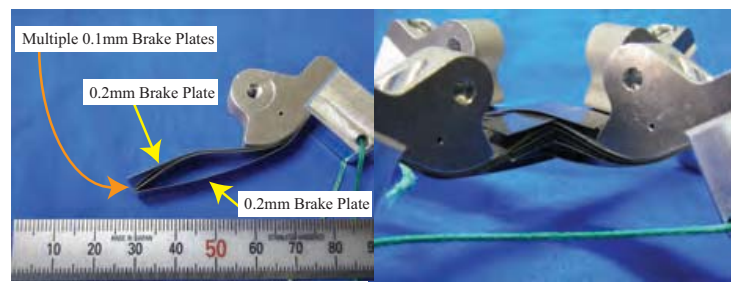

Fig. 12. Multiple brake sheets

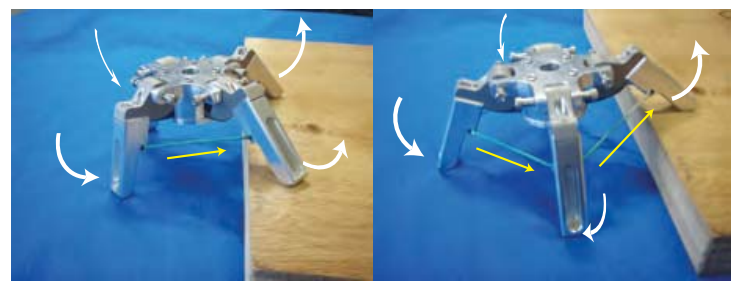

Fig. 13. Deformation mechanism

the center rod, using the friction of the brake sheet. This mechanism can generate sufficient braking force using the weight of the robot regardless of the number of fingers. Fixing force can be increased by increasing the number of the brake sheet, and the finger mechanism can be fixed at arbitrary angle because the brake plate moves according to motion of the finger mechanism. The brake sheets are composed of multiple brake sheets of $0.1 \mathrm{~mm}$ in thickness sandwiched by the brake sheets of $0.2 \mathrm{~mm}$, and this method makes multiple brake sheets to move according to the motion of the finger mechanism by using the brake sheet of $0.2 \mathrm{~mm}$ as a guide (Fig.12).

The slide mechanism to operate the shape-fixing mechanism is composed of pipes and four rods. The inside of pipes guide four rods and are Tufram processed to reduce the friction of the sliding. This structure achieves the small backslash and the solid composition after it fixed (Fig.11). 


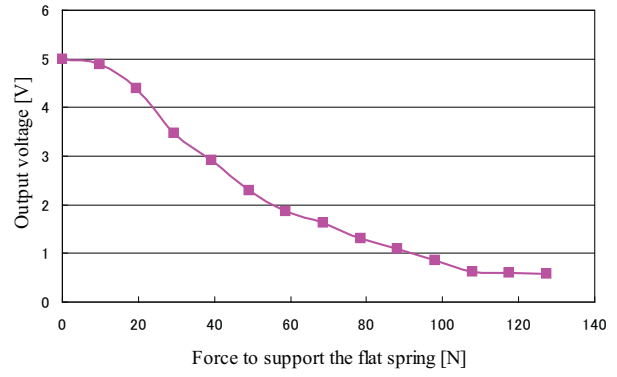

Fig. 14. Relation between load and output of support force sensor

\section{Vertical Force Sensor}

The stability can be improved by installing the force sensor which permits the robot to detect walking environment and adjust the gait depending on the distribution of the vertical force acting on each foot.

The vertical force sensor system is composed of an optical sensor and the leaf spring (Fig.9 and Fig.10). The optical sensor is composed of a photo interrupter and a shading $\operatorname{rod}($ EE-SA113 OMRON), and can change the output of internal photo interrupter by moving the actuator part. The basic principle is as follows; when the force is applied to the rod, the rod slightly lifts the leaf spring. And, leaf spring pushes the shading rod which is installed in the micro photo sensor, and the force is measured by reading the change of the output of the sensor.

This force sensor can compose the sensor system that an external amplifier is unnecessary, cheap, and compact. Moreover, the measurement range of the force can be changed by designing the leaf spring. The result of the relation between the output and the load is shown in Fig.14. From this result, it is confirmed that the support force sensor is able to measure the load within the range of about $100 \mathrm{~N}$.

\section{EXPERIMENTS}

The terrain adaptive sole is installed in TITAN VIII and walking experiments were conducted.

\section{A. Walking Experiments on Terrain Including Small Bump}

Walking experiments were conducted on the terrain where the height differences are $20 \mathrm{~mm}$, which is relatively small compared with the size of the robot. TITAN VIII moved at a static trot gait so that the projection of gravity was moving inside the support polygon formed by a pair of the diagonal feet (speed $0.071 \mathrm{~m} / \mathrm{s}$ and $2.1 \mathrm{~s} / \mathrm{step}$ ). At static trot gait, because quadruped walking robot uses only two feet and the support polygon is formed by two sole area, if the foot mechanism can not generate the torque to prevent from falling, support polygon will be too small to walk statically. And, three-axes acceleration and two-axes angular velocity (around $\mathrm{x}$ axis and $\mathrm{y}$ axis ) were measured by the attitude sensor (ADXL330 Analog Devices, IDG300 InvenSense) which is installed at TITAN VIII (Fig.15).

The overview of the walking experiment with the terrain adaptive sole is shown in Fig.18 and Fig.19, and the overview

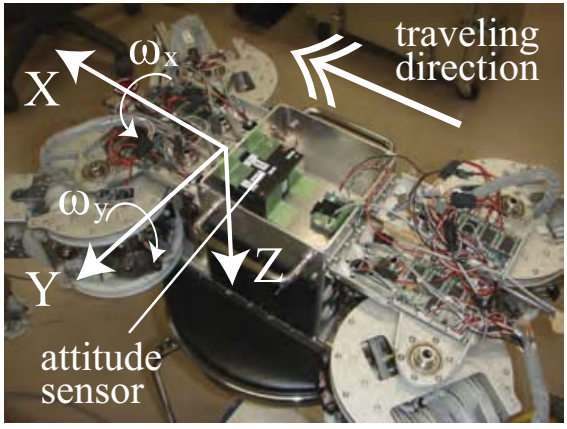

Fig. 15. Direction of attitude sensor

of the walking experiment with the flat hard sole is shown in Fig.16 and Fig.17. The Figs show that the terrain adaptive sole can adapt to the bump, while the flat hard sole lost the balance and slipped down from the bump. And, Fig.20 and Fig.21 show the output of the attitude sensor with each sole mechanism. Though, from Fig.20, TITAN VIII with flat hard sole was subjected to impacts caused by slippage, it is confirmed from Fig.21 that TITAN VIII with terrain adaptive sole could walk stably on the ground.

We carried out this walking experiment in ten times with each sole mechanism. The mean value of the peak value measured by the posture sensor is shown in Table.II. We can observe that the values of the experiments with the terrain adaptive sole are lower at all the values than with the flat hard sole, suggesting that the terrain adaptive sole achieved a stable walking and successfully negotiates relatively small ruggedness by its passive mechanism.

\section{B. Walking Experiment on Terrain Including Variety of Bumps}

A relatively large height difference also can be adapted to by vertical force sensor. We conducted the walking experiment on the rough terrain with a variety of ruggedness types included. The terrain which is composed of random shape stones which are about $200 \mathrm{~mm}$ in the diameter and lumber with difference of $30 \mathrm{~mm}$ or less was produced as rough terrain, and TITAN VIII walked on the terrain at crawl gait(speed $0.077 \mathrm{~m} / \mathrm{s}$ and $3.92 \mathrm{~s} / \mathrm{step}$ ). In this experiment, TITAN VIII walked using the vertical force sensor to detect relatively large height difference and keeping the height of the body at the same level by stopping the landing motion of the leg when the developed sole mechanism touched the ground. Leg trajectory can be divided into 4 sections; lifting up, moving forward, touching the ground and moving backward. In touching down phase, the leg motion stops when the force sensor detects the vertical reaction force.

Fig.22 shows the walking sequence of the experiment. It was confirmed that the terrain adaptive sole permits the walking robot to walk stably adapting to such a complex rough terrain by using a very simple strategy for a gait generation. 
TABLE II

MEAN PEAK VALUE OF EACH SOLE MECHANISM

\begin{tabular}{|c|c|c|c|c|c|}
\hline & $\begin{array}{c}\mathrm{X} \text { axis acceleration } \\
\left(\mathrm{m} / \mathrm{s}^{2}\right)\end{array}$ & $\begin{array}{c}\mathrm{Y} \text { axis acceleration } \\
\left(\mathrm{m} / \mathrm{s}^{2}\right)\end{array}$ & $\begin{array}{c}\mathrm{Z} \text { axis acceleration } \\
\left(\mathrm{m} / \mathrm{s}^{2}\right)\end{array}$ & $\begin{array}{c}\mathrm{X} \text { axis angular velocity } \\
(\mathrm{rad} / \mathrm{s})\end{array}$ & $\begin{array}{c}\mathrm{Y} \text { axis angular velocity } \\
(\mathrm{rad} / \mathrm{s})\end{array}$ \\
\hline flat hard sole & 3.14 & 5.09 & 6.04 & 0.39 & 0.61 \\
\hline terrain adaptive sole & 2.65 & 4.27 & 3.64 & 0.30 & 0.47 \\
\hline
\end{tabular}

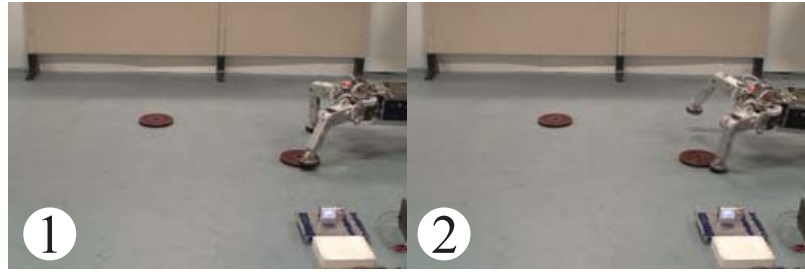

Fig. 16. Walking experiment with flat hard sole on small bump

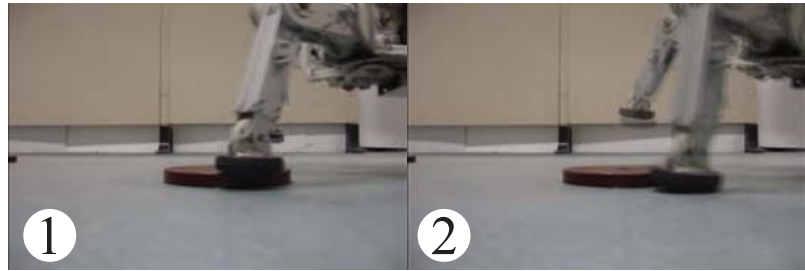

Fig. 17. Flat hard sole on small bump

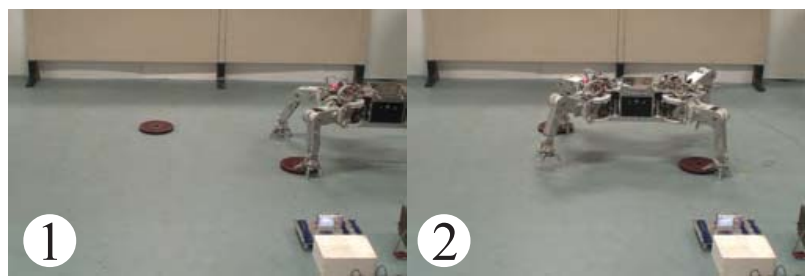

Fig. 18. Walking experiment with terrain adaptive sole on small bump

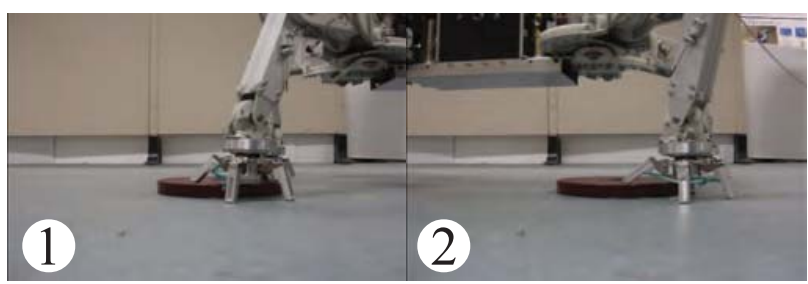

Fig. 19. Terrain adaptive sole on small bump

\section{Walking Experiments on an Outdoor Environment}

We carried out walking experiments in a real outdoor environment to evaluate its ability. Fig.23 and Fig.24 show the overview of experimental results of the static trot walking (speed $0.071 \mathrm{~m} / \mathrm{s}$ and $2.1 \mathrm{~s} / \mathrm{step}$ ) on the unpaved road and the gravel, and the crawl gait(speed $0.077 \mathrm{~m} / \mathrm{s}$ and $3.92 \mathrm{~s} / \mathrm{step}$ ) on bumps and slopes, respectively. Figures show that the terrain adaptive sole can adapt to the terrain and permits the walking robot to walk stably.

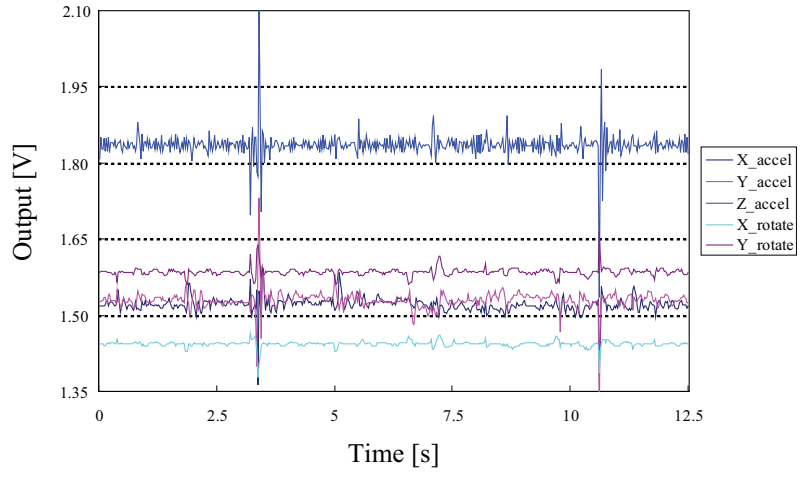

Fig. 20. Output of attitude sensor with flat hard sole

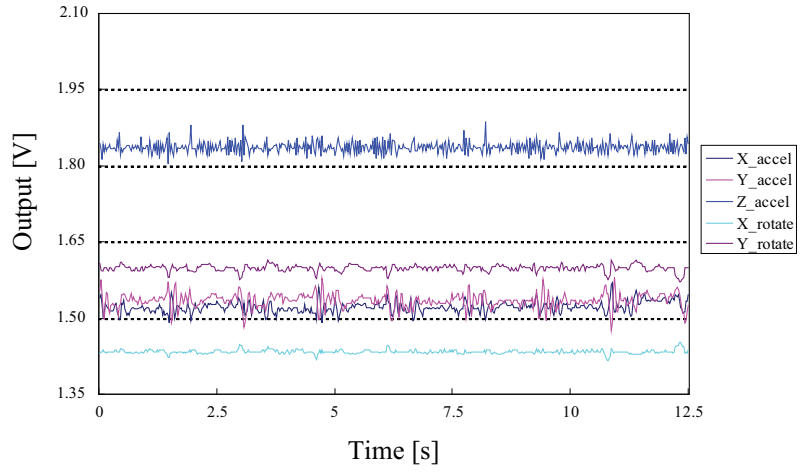

Fig. 21. Output of attitude sensor with terrain adaptive sole

\section{CONCLUSIONS}

In this paper, we described the development of the terrain adaptive sole to secure a steady foothold of the multi-legged walking robot on rough terrain. As a composition of the terrain adaptive sole, the deformation mechanism which can deform according to the terrain, and the shape-fixing mechanism which can fix the sole shape after deformation, and the vertical force sensor were developed. Moreover, terrain adaptive sole was installed in TITAN VIII, the experiment that walked on rough terrain was conducted showing the effectiveness to increase the stability.

It will be necessary to optimize the design of the terrain adaptive sole. Concretely, the number of braking sheets and the property of the leaf spring should be discussed. In this paper, we do not focus on a soft deformable environment such as a soil road in a natural environment. Decreasing the foot pressure in order not to deform or damage to the ground 


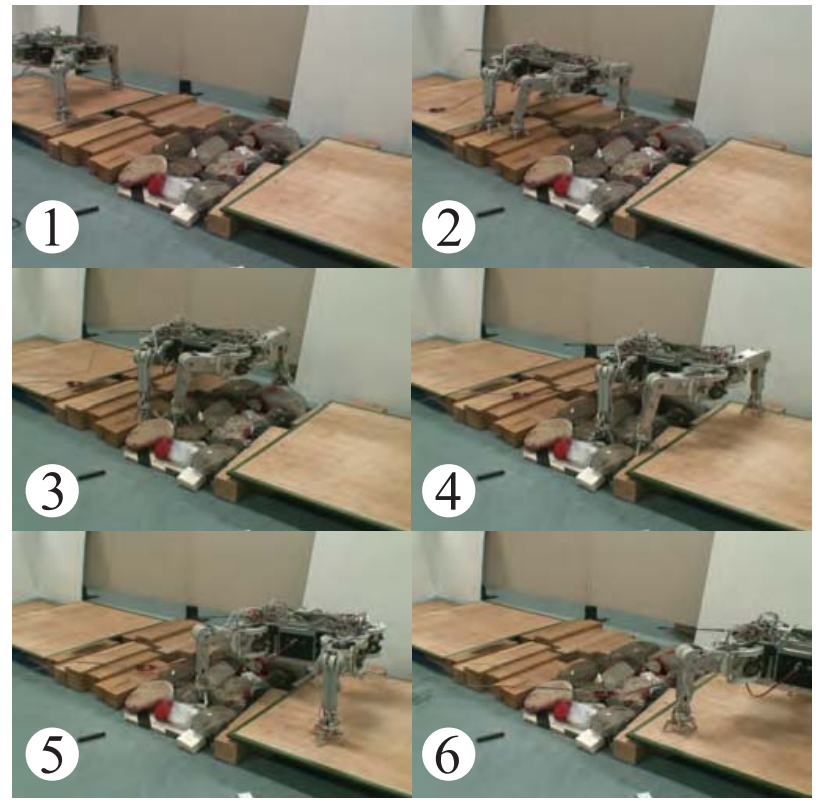

Fig. 22. Walking experiment on rough terrain

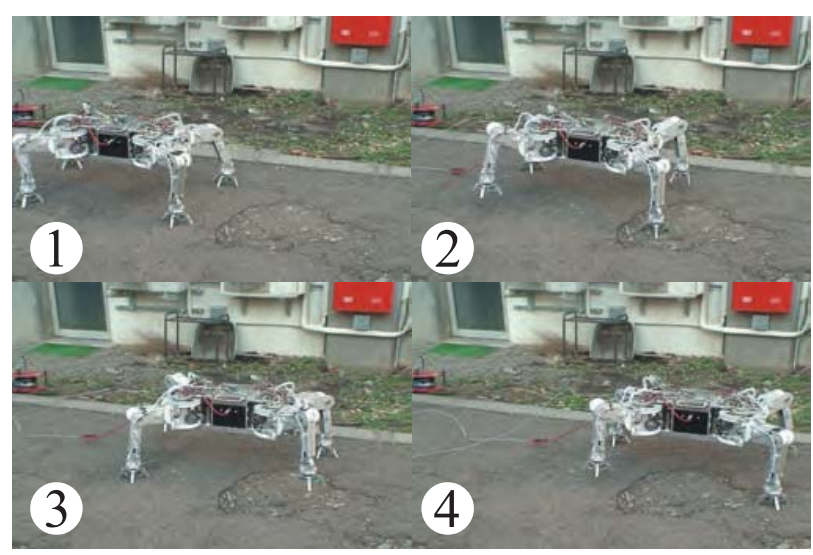

Fig. 23. Walking on unpaved road

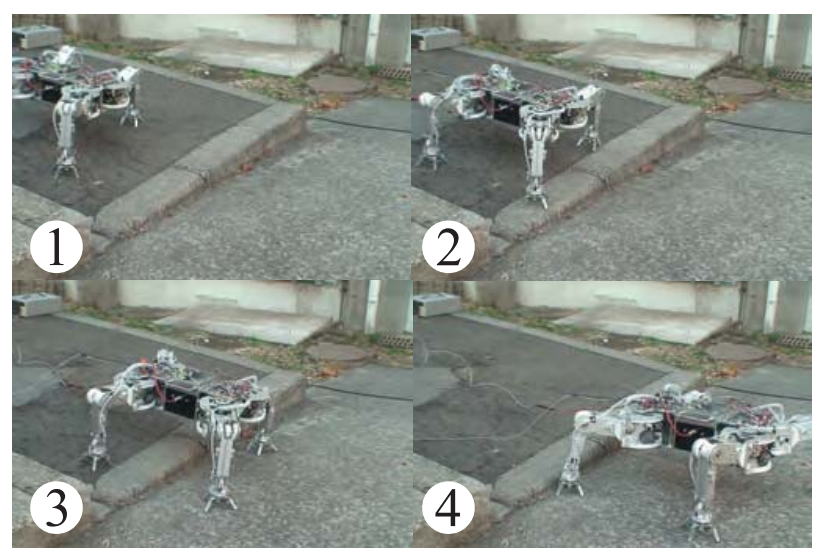

Fig. 24. Traversing step in outdoor environment forms an important part of our future work.To solve these problems, covering the entire mechanism with a elastic sheet such as rubber may be a promising solution.

\section{ACKNOWLEDGMENT}

The authors would like to thank Frederic Chucholowski for a development of a useful low level controller of TITAN VIII which remarkably helps us in hardware experiments.

\section{REFERENCES}

[1] K. Kato, S. Hirose : Development of Quardruped Walking Robot, TITAN-IX -Mechanical Design Concept and Application for the Humanitarian Demining Robot-, Advanced Robotics, 15, 2, pp. 191204, 2001

[2] R. Hodoshima, T. Doi, Y. Fukuda, S. Hirose, T. Okamoto, and J. Mori: Development of a quadruped walking robot TITAN XI for steep slope operation step over gait to concrete frames on steep slopes, J. Robot. Mechatron., vol. 1, no. 19, pp. 13-26, 2007.

[3] S. Hirose, K. Yoneda. H. Tsukagoshi: TITAN VII : Quadruped Walking and Manipulating Robot on a Steep Slope, Proc. ICRA, pp. 494-500, 1997.

[4] M. Ogata, S. Hirose: Study on Ankle Mechanism for Walking Robots - Fundamental Considerations on it Functions and Morphology -,J. of Robotics and Mechatronics,16,1,pp. 23-30, 2004

[5] K. Hashimoto, Y. Sugahara,et al.: Walking Technology Adaptable to Uneven Terrain in Real Environment for Biped Locomotors, Proc. IEEE/RSJ International Conference on Intelligent Robots and Systems,pp. 1755-1760,2006.

[6] K. Arikawa, S. Hirose: Development of Quadruped Walking Robot TITAN-VIII,Proc. of IROS, pp. 208-21 4, 1996.

[7] K. Nonami, N. Shimoi, Q. J. Huang, D. Komizo, H. Uchida: Development of Teleoperated Six-legged Walking Robot for Mine Detection and Mapping of Mine Field, Proc. of the 2000 IEEE/RSJ Int.Conf. on Intelligent Robots and Systems, pp. 775-779, 2000.

[8] S. Hirose: Connected Differential Mechanism and its Applications ROBOT GRIPPERS, IFS, pp. 141-153, 1986. 